\title{
Ammonia Removal by Metabolic Scavengers for the Prevention and Treatment of Hepatic Encephalopathy in Cirrhosis
}

\author{
Roger F. Butterworth ${ }^{1}$ (D)
}

Accepted: 26 March 2021 / Published online: 22 April 2021

(c) The Author(s) 2021

\begin{abstract}
Effective lowering of circulating ammonia is the mainstay strategy in the prevention and treatment of hepatic encephalopathy in cirrhosis and there is increasing interest in agents with the metabolic potential for the active removal of ammonia by the liver and skeletal muscle by agents including L-ornithine L-aspartate, branched-chain amino acids, as well as the re-purposing of benzoate and phenylacetate currently employed for the control of hyperammonaemia in congenital urea-cycle enzymopathies. Based upon results of multiple systematic reviews with meta-analyses, L-ornithine L-aspartate demonstrably lowers circulating ammonia in patients with cirrhosis with concomitantly improved mental status. Distinct mechanisms responsible include optimisation of hepatic metabolic pathways for ammonia removal as well as direct hepatoprotective effects involving the release of glutathione and of nitric oxide with beneficial effects on hepatic microcirculation. L-ornithine L-aspartate also prevents cirrhosis-related sarcopenia, leading to increased capacity for ammonia removal by skeletal muscle. Branched-chain amino acids continue to be prescribed as nutritional supplements with the potential to result in improvements in liver function. Sodium benzoate, glycerol phenylbutyrate and an analogous compound L-ornithine phenylacetate were also evaluated. Glycerol phenylbutyrate was the only agent with a beneficial effect on both hyperammonaemia and hepatic encephalopathy. None were superior to lactulose for the lowering of blood ammonia.
\end{abstract}

\section{Introduction}

Hepatic encephalopathy (HE) in cirrhosis is a severe neuropsychiatric complication characterised clinically by a spectrum of alterations of personality, motor coordination and cognitive function with a major impact on health-related quality of life [1], progressing from an inability to perform simple tasks to stupor and coma [2]. A consensus of evidence supports the notion that increased concentrations of circulating and brain ammonia resulting from the cirrhotic liver's inability to effectively remove it in the form of urea or glutamine is the principal cause of HE in cirrhosis [3].

\section{Hepatic Ammonia Removal and the Concept of Ammonia Scavenging}

Excess ammonia generated principally by protein digestion in the gut is carried to the liver by the portal vein. Removal of

Roger F. Butterworth

rb@enceph.com

1 Department of Medicine, University of Montreal, 45143

Cabot Trail, Englishtown, NS B0C 1H0, Canada

\section{Key Points}

The effective lowering of circulating ammonia remains the mainstay strategy for the prevention and treatment of hepatic encephalopathy in liver cirrhosis. Agents used for this purpose include mixtures of essential (branched chain) or non-essential (L-ornithine L-aspartate) amino acids. Efficacy of the latter mixture relies on the stimulation of metabolic ammonia-lowering pathways and on the reduction of sarcopenia and hepatoprotective actions

Alternative approaches to the above are provided by the re-purposing of agents such as benzoate, phenylacetate and their analogues that are well-established agents for the treatment of urea cycle enzymopathies

Results of systematic reviews and meta-analyses of controlled clinical trials confirm that only L-ornithine L-aspartate and glycerol phenylbutyrate are effective for the lowering of circulating ammonia, leading to an improved mental state in patients with cirrhosis and hepatic encephalopathy. L-Ornithine L-aspartate is effective for hepatic encephalopathy prophylaxis in these patients 
excess ammonia by the liver makes use of two independent mechanisms involving specific cell types in the liver acinus characterised by distinct metabolic processes. Incorporation of ammonia into the urea molecule takes place in periportal hepatocytes known to express genes associated with the constituent enzymes of the urea cycle, whereas any remaining ammonia is removed by incorporation into the molecule of glutamine via the enzyme glutamine synthetase located in perivenous hepatocytes. This latter step is frequently referred to as "scavenging" of ammonia by the liver. The location of these systems in relation to the well-established "inter-organ transport or "trafficking" of ammonia is depicted in a simplified schematic in Fig. 1a [3]. More recently, the notion of ammonia scavenging is increasingly being employed to signify ammonia removal by mechanisms other than urea synthesis such as glutamine synthesis by perivenous hepatocytes but also by other organs such as skeletal muscle.

In cirrhosis, loss of hepatic parenchyma leads to increased vascular resistance and portal hypertension that, in turn, may result in portal-systemic shunting of ammonia-rich venous blood. Concomitantly, significant losses of up to $85 \%$ of functional periportal and perivenous hepatocytes occur, resulting in severe impairment of removal of ammonia and sustained hyperammonaemia [3] (Fig. 1b).

The lowering of circulating ammonia continues to be the mainstay strategy for the prevention and treatment of $\mathrm{HE}$ in cirrhosis. Agents with demonstrated efficacy for the reduction of hyperammonaemia in the context of cirrhosis and $\mathrm{HE}$ fall into one of two major categories.

Group [1] agents with the ability to reduce ammonia absorption from the gut (includes non-absorbable disaccharides [lactulose, lactitol], antibiotics [aminoglycosides, rifaximin], probiotics, purgatives such as polyethylene glycol3350-electrolyte solutions [4] and a carbon microsphere preparation AST-120) [5].

Group [2] agents have the metabolic potential for the active removal of ammonia by the liver and other organs such as skeletal muscle. Such agents comprise mixtures of essential or non-essential amino acids including L-ornithine L-aspartate (LOLA) and branched-chain amino acids (BCAAs). In addition, the re-purposing of agents or their analogues that are currently used for reducing hyperammonaemia in patients with congenital urea-cycle enzymopathies (sodium benzoate, phenylacetate and analogues) is under investigation.

Both classes of agents have been employed for the lowering of circulating ammonia in patients with cirrhosis and in most (but not all) cases this has resulted in concomitant improvements in mental state and/or grade of HE. The present review focusses on the second group of agents for which the efficacy for ammonia lowering and mental state improvements are compared with other Group 2 agents and where results are available, have been compared with the efficacy of agents from Group 1 such as lactulose.

\section{LOLA}

L-Ornithine L-aspartate is a 1:1 stable salt of the non-essential naturally-occurring amino acids L-ornithine and L-aspartate [6]. There are three independent mechanisms whereby LOLA treatment has the capacity to result in the improvement of mental state as a result of the lowering of circulating ammonia in patients with cirrhosis [7].
Fig. 1 Simplified schematic of the steps involved in inter-organ trafficking of ammonia between the gut, liver, skeletal muscle, brain and kidney in normal individuals (a) compared to patients with cirrhosis and hepatic encephalopathy (b)
A

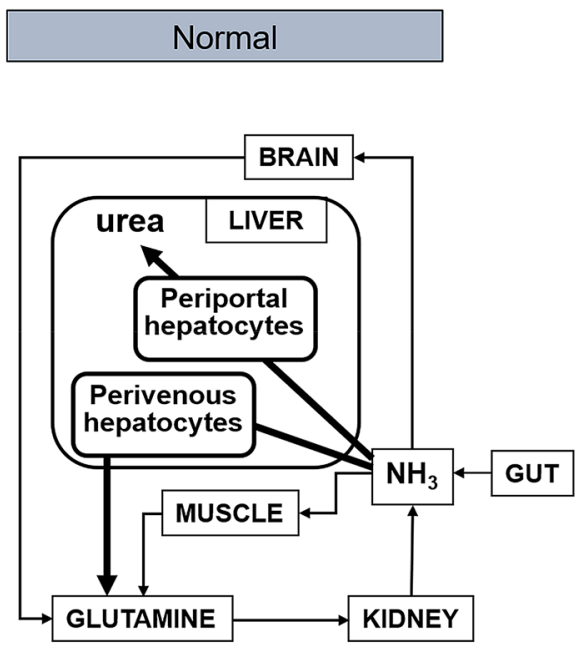

B
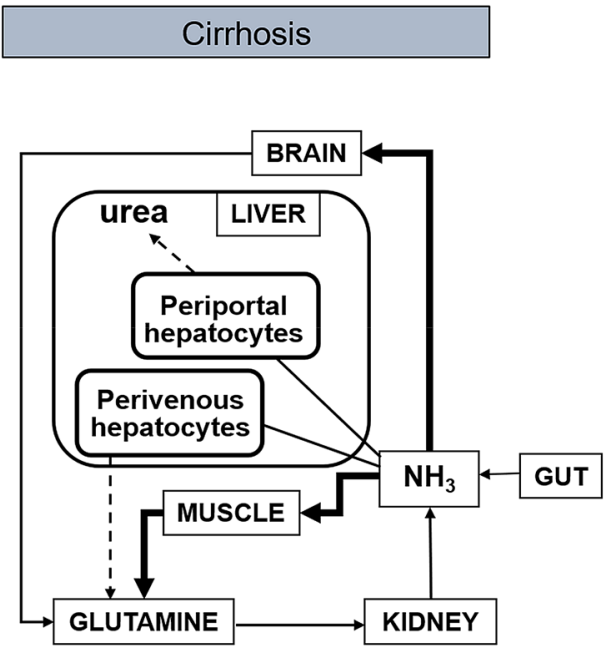


\subsection{Optimisation of Metabolic Pathways Responsible for Ammonia Removal by Residual Periportal and Perivenous Hepatocytes}

Studies in isolated hepatocytes reveal that urea synthesis from ammonia is limited by the supply of L-ornithine. Furthermore, the requirements for L-ornithine for urea synthesis are increased as a function of the supply of ammonia [7]. L-Ornithine, being a urea cycle intermediate and activator of the urea cycle enzyme carbamoyl phosphate synthetase, has the capacity to result in increased urea synthesis, a process that occurs in the residual $15-20 \%$ of functional periportal hepatocytes in the cirrhotic liver [8].

Furthermore, both L-ornithine and L-aspartate are substrates for transamination reactions resulting in the increased synthesis of glutamate (the obligate substrate for glutamine synthetase), leading to increased production of glutamine in perivenous hepatocytes, skeletal muscle and brain. Increased brain glutamine signals have been reported in magnetic resonance spectroscopic investigations of patients with cirrhosis and $\mathrm{HE}$ where the severity of the HE grade was positively correlated with glutamine signal strength [9].

\subsection{Direct Hepatoprotective Effects of LOLA}

Direct evidence of a hepatoprotective effect of treatment of patients with cirrhosis and HE is provided in reports of several randomised controlled trials (RCTs) in which treatment with LOLA had resulted in lowering of circulating ammonia together with improvements of mental function. Evidence of improvements of hepatic function post-LOLA included significant attenuation of transaminases and decreases of serum bilirubin together with improvements of prothrombin times [10] and improvements in Child-Pugh and MELD scores [11]. Similar indices of hepatoprotection by LOLA were also reported in patients with cirrhosis and post-transjugular intrahepatic portosystemic shunt encephalopathy [12]. A review of potential mechanisms implicated in these hepatoprotective properties of LOLA suggested that the accumulation of the antioxidant glutathione in addition to the increase in synthesis of nitric oxide with consequent beneficial effects on hepatic microcirculation [13]. L-Ornithine L-aspartate may also exhibit hepatoprotective effects in patients with non-alcoholic fatty liver disease [14].

\subsection{Prevention of Cirrhosis-Related Sarcopenia (Muscle Wasting), Resulting in Optimisation of Ammonia Removal by Skeletal Muscle}

Arterio-venous difference studies together with studies of the dynamics of ammonia metabolism using ${ }^{13}-\mathrm{NH}_{3}$ positron emission tomography in patients with cirrhosis reveal that skeletal muscle plays an important role in the process of ammonia removal in patients with cirrhosis, a process that appears to be catalysed by a post-translational induction of the glutamine synthetase gene [15]. Severe muscle wasting (sarcopenia) is a common complication of cirrhosis that is associated with poor prognosis, poor post-transplant outcomes and increased mortality. Moreover, the fractional extraction of ammonia is significantly decreased in cirrhotic patients with sarcopenia and, to make matters worse, there is convincing new evidence to suggest that ammonia per se causes damage to myotubes resulting in reduced muscle strength [16]. Based upon these reports, it was suggested that a "vicious cycle" exists in cirrhosis whereby hyperammonaemia resulting from impaired hepatic ammonia removal has the potential to result in muscle dysmetabolism, autophagy and sarcopenia, resulting in the inability of muscle to effectively fulfil its role as alternate pathway for ammonia removal [17]. This, in turn, leads to the worsening of hyperammonaemia and the cycle continues as shown in Fig. 2a, b.

Studies in an experimental animal model of chronic liver failure demonstrate that treatment with LOLA and an antibiotic is effective for the reduction of circulating and muscle ammonia, resulting in improved muscle function and fracture of the vicious cycle. These findings provide a third mechanism whereby LOLA has the potential to reduce hyperammonaemia and HE severity in cirrhosis [7].

\subsection{Evidence Base for the Efficacy of LOLA for the Lowering of Blood Ammonia and Treatment of $\mathrm{HE}$ in Cirrhosis}

Results of three independent systematic reviews with a metaanalysis addressing the issue of the efficacy of LOLA for the treatment of HE in cirrhosis confirm that the intravenous and oral formulations of LOLA are equally effective for the lowering of blood ammonia in patients with cirrhosis with concomitant improvements of mental state (Table 1). In the first review, the results of eight RCTs in 709 patients with cirrhosis and $\mathrm{HE}$ revealed significant lowering of blood ammonia [mean difference (MD) $-17.50,95 \%$ confidence interval (CI) $-27.73,-7.26, Z=3.35, p<0.0008$ ] (Fig. 2a) with concomitant significant improvements in mental state (relative risk (RR) 1.36, 95\% CI 1.10, 1.69, $Z=2.82, p$ $<0.005$ ) (Fig. 2b) [18, 19]. Both the oral and intravenous formulations of LOLA were effective for ammonia lowering and for the improvement of mental state in patients with cirrhosis and minimal HE (MHE) or overt HE (OHE) (Table 1).

Results of a subsequent Cochrane review and meta-analysis of 22 RCTs for a total of 1375 patients largely confirmed the efficacy of LOLA for the improvement of hyperammonaemia and mental state grade [20]. However, the quality of the evidence in this analysis was brought into question because the study had included data from a large number of 
abandoned and incomplete trials for which it was not possible to assess the risk of bias using conventional tools. This led the authors to assign scores of very low quality despite highly significant indications of efficacy of LOLA (RR 0.70 , 95\% CI 0.59, 0.83, $Z=4.01, p<0.000061)$. The conclusions based on the results of the analysis of the trials taken together were judged to be "uncertain". However, when a sub-group analysis was performed either for completed trials or published trials in this series, significant improvements became apparent both for ammonia lowering and for improvement of mental state as summarised in Table 1 . The magnitude and direction of change were largely confirmatory of the changes reported in previous reports $[18,19]$ as summarised in Table 1.

A third systematic review of the efficacy of LOLA vs placebo/no treatment involved the extraction of data from five RCTs and 233 patients with cirrhosis and MHE. Again, significant improvements of hyperammonaemia and mental state were noted with OR 3.19; 95\% CI 1.44, 7.11, $Z=2.85$, $p<0.004$ and quality of evidence scored as moderate. Furthermore, the development of OHE in 104 of these patients with MHE in two RCTs in this analysis was reduced by LOLA compared to placebo/no intervention with OR 0.11: 95\% CI $0.02,0.59, Z=2.56, p<0.01[21]$.

In a novel extension of the notion of a protective effect of LOLA against the progression to OHE in patients with cirrhosis, a subsequent systematic review with a meta-analysis demonstrated for the first time that LOLA is effective for the prevention of OHE across a wide range of clinical presentations. To be precise, LOLA was shown to be effective both

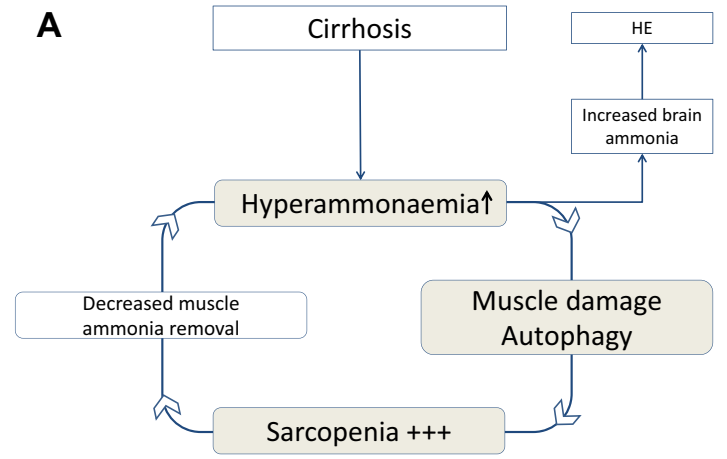

Fig. 2 a Schematic of the vicious cycle whereby hyperammonaemia resulting from decreased ammonia removal by the liver leads to muscle damage/autophagy and sarcopenia. Sarcopenia results in a serious diminution of the capacity of muscle to remove blood-borne ammonia leading to worsening of hyperammonaemia and the vicious cycle continues. b Schematic of the vicious cycle whereby treatment for primary or secondary OHE prophylaxis and also for the slowing of the progression from MHE to OHE in cirrhosis and for the prevention of OHE occurring post-transjugular intrahepatic portosystemic shunt [22]. These findings are summarised collectively in Fig. 3c.

\section{BCAAs}

During the late 1970s and 1980s, a great deal of emphasis was placed upon studies of the possible beneficial effects of the treatment of patients with cirrhosis and HE with mixtures of BCAAs. The rationale for their use was initially based upon the hypothesis that BCAA treatment would normalise disordered patterns of distribution of aromatic/BCAA ratios and that, in so doing, would prevent the accumulation of a series of trace amines, which were thought to act as "false neurotransmitters" that contribute to the pathogenesis of HE in cirrhosis [26, 27]. Early controlled clinical trials revealed a benefit of BCAAs in patients with MHE [28] but not in OHE [29]. This difference of opinion has continued to the present time with reports of efficacy of BCAAs for the lowering of blood ammonia and improvement of mental state [30] vs little by way of change [31]. The "false neurotransmitter hypothesis" for the pathogenesis of HE in cirrhosis is no longer tenable. However, BCAAs continue to be prescribed as nutritional supplements with the potential to result in improvements in liver function, health-related quality of life and reduced hospitalisation rates in patients with advanced cirrhosis [32].

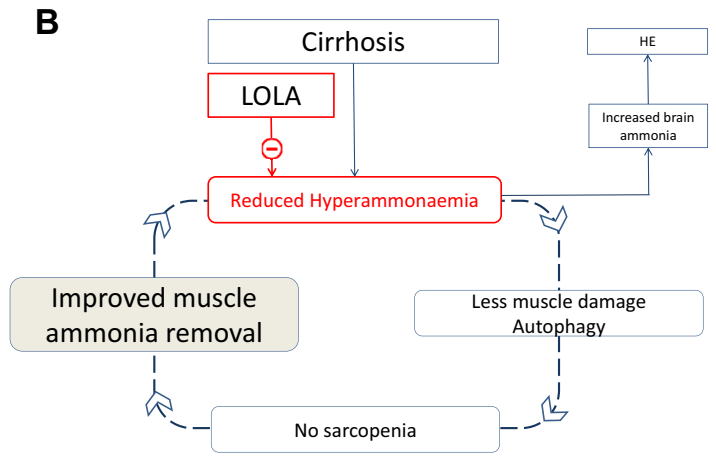

with L-ornithine L-aspartate (LOLA) results in the lowering of hyperammonaemia by multiple mechanisms described in the text, which, in turn, relieves the damage to skeletal muscle/sarcopenia and the muscle's capacity to remove blood-borne ammonia is restored. $H E$ hepatic encephalopathy 
Table 1 Evidence for the efficacy of LOLA for HE in cirrhosis: results of three systematic reviews/meta-analyses

Butterworth et al. [18]

10 RCTs published, 848 patients

MHE or OHE

LOLA iv or oral formulation vs placebo/no intervention, high quality

Results for all types of HE: RR 1.36, 95\% CI 1.10, 1.69, $Z=2.82(p=0.005)$

Results: effective for MHE: RR 2.15, 95\% CI 1.48, 3.14, $Z=3.98(p<0.0001)$

Results: effective for OHE: RR 1.19, 95\% CI 1.01, 1.39, $Z=2.14(p=0.03)$

Goh et al. [20]

29 RCTs some published, some completed, 1891 patients

LOLA iv or oral formulation vs placebo/no intervention, low quality

Results of all trials effective for HE: RR 0.70, 95\% CI 0.59, 0.83, $Z=4.01(p=0.000061)$

Results of completed trials, effective for all types of HE (12 RCTs, 994 patients): RR $0.53,95 \%$ CI $0.48,0.83, Z=3.26$ ( $p=0.0011)$

Results of published trials, effective for all types of HE (12 RCTs, 1032 patients): RR $0.65,95 \%$ CI $0.50,0.85, Z=3.13$ ( $p=0.0017$ )

Dhiman et al. [21]

5 RCTs published, 233 patients

LOLA iv or oral formulation vs placebo/no intervention, moderate quality

Results: LOLA is effective for lowering of hyperammonaemia and for reversal of MHE: OR 3.19, 95\% Cl 1.44, 7.11, $Z=2.85(p<0.004)$

Results: LOLA slows rate of development of OHE [2 trials, 104 patients] with OR $0.11,95 \%$ CI $0.02,0.59, Z=2.56(p<0.01)$

$C I$ confidence interval, $i v$ intravenous, $L O L A$ L-ornithine L-aspartate, $M H E$ minimal hepatic encephalopathy, $O H E$ overt hepatic encephalopathy, $O R$ odds ratio, $P r I$ prediction interval, $R C T$ randomised controlled trial, $R R$ relative risk

\section{Repurposing of Ammonia-Lowering Agents Used to Treat UCDs for the Treatment of HE in Cirrhosis}

The treatment of inborn errors of urea synthesis relies on the activation of alternative metabolic pathways for waste nitrogen synthesis and excretion [33]. In this regard, a group of substances including sodium benzoate, sodium phenylacetate and a range of their analogues is currently used for the effective lowering of blood ammonia in urea cycle enzymopathies, a group of disorders principally of children characterised by severe neurological impairment that may progress to include seizures and mental retardation. Despite the high sodium load associated with some of these preparations [34], recent years have seen increased interest in repurposing of these agents for the management of HE in cirrhosis as follows.

\subsection{Sodium Benzoate}

Results of an RCT demonstrated that sodium benzoate was equivalent in efficacy to lactulose for both the lowering of arterial ammonia and for improvement of mental state in patients with cirrhosis and acute HE [35]. A subsequent analysis of the same data as part of a Cochrane systematic review however revealed no beneficial or harmful effects of sodium benzoate compared to non-absorbable disaccharides with respect to mortality, grade of HE or lowering of blood ammonia [36].

\subsection{SPA, SPB}

Sodium phenylacetate (SPA) has been successfully employed for the treatment of hyperammonaemia related to an inborn error of the urea cycle [37] and sodium phenylbutyrate (SPB) has been approved in the USA and in Europe for the treatment of a range of chronic urea cycle disorders where its mechanism of action is predicated on its ability, following oxidation to SPA, to lower ammonia by the enhancement of the excretion of waste nitrogen primarily in the liver and kidney in the form of phenylacetylglutamine [38]. Given the prime importance of hepatic and renal systems for their mechanisms of anti-hyperammonemic actions, it is not clear whether chronic liver disease often associated with renal impairments impacts the efficacy of conversion of SPA or SPB to phenylacetylglutamine. This issue needs to be carefully addressed. Neither SPA nor SPB has been widely used for the prevention or treatment of HE in cirrhosis. A range of common adverse effects affecting the respiratory, lymphatic and nervous systems by some preparations containing phenylacetate/phenylbutyrate has been described [34].

\subsection{Glycerol Phenylbutyrate}

A more recent addition to the available agents for the treatment of hyperammonaemia resulting from urea cycle disorders (UCDs) is glycerol phenylbutyrate (GPB). Like SPA, it contains phenylbutyrate, a pro-drug that is readily 


\section{A - Efficacy of LOLA versus placebo/no intervention: $\mathrm{NH}_{3}$ lowering (all $\mathrm{HE}$ trials)}

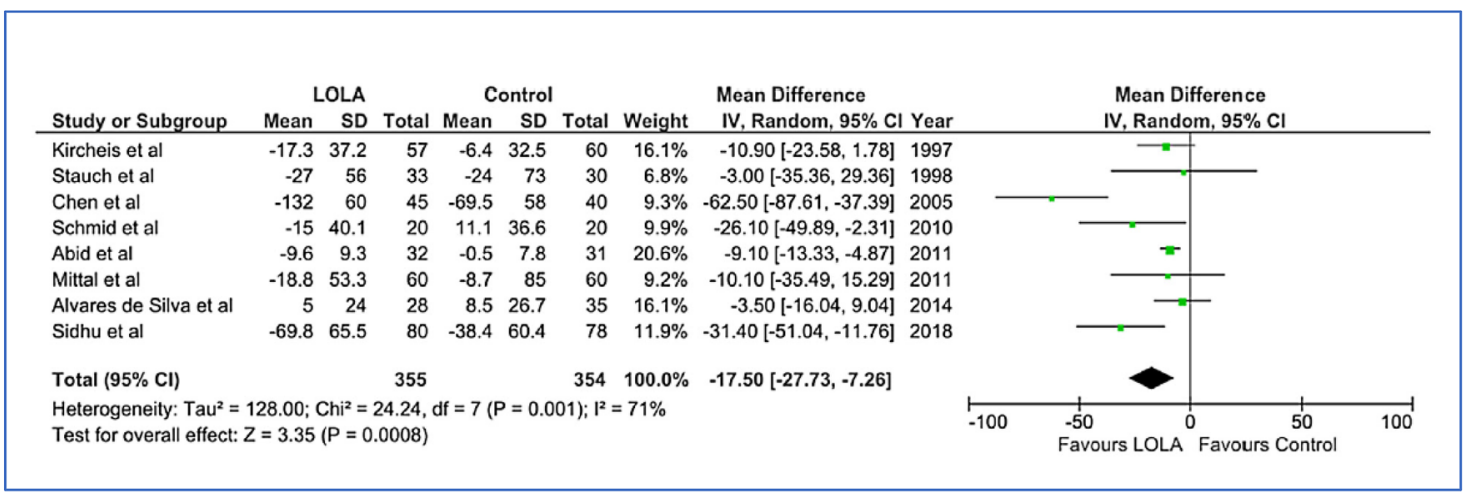

Forest plot indicating the pooled effect of LOLA versus placebo/no intervention for the lowering of blood ammonia [19]. Abbreviations: RR: Risk Ratio, Cl: Confidence interval, SD: standard deviation.

\section{B - Efficacy of LOLA versus placebo/no intervention: Mental State (all HE trials)}

\begin{tabular}{|c|c|c|c|c|c|c|c|c|c|c|c|}
\hline \multirow{2}{*}{$\begin{array}{l}\text { Study or Subgroup } \\
\text { Kircheis et al }\end{array}$} & \multicolumn{2}{|c|}{$\begin{array}{l}\text { LOLA } \\
\text { Events Total }\end{array}$} & $\begin{array}{l}\text { Contro } \\
\text { Events }\end{array}$ & $\begin{array}{l}\text { ol } \\
\text { Total }\end{array}$ & Weight & \multicolumn{2}{|c|}{$\begin{array}{l}\text { Risk Ratio } \\
\text { M-H, Random, } 95 \% \mathrm{CI} \text { Year }\end{array}$} & \multicolumn{4}{|c|}{$\begin{array}{c}\text { Risk Ratio } \\
\text { M-H, Random, } 95 \% \mathrm{Cl}\end{array}$} \\
\hline & 37 & 63 & 20 & 63 & $11.2 \%$ & $1.85[1.22,2.81]$ & 1997 & & & -- & \\
\hline Stauch et al & 17 & 34 & 8 & 32 & $6.5 \%$ & $2.00[1.01,3.98]$ & 1998 & & & $\longrightarrow$ & \\
\hline Chen et al & 43 & 45 & 33 & 40 & $17.4 \%$ & $1.16[0.99,1.35]$ & 2005 & & & $=$ & \\
\hline Ahmad et al & 37 & 40 & 31 & 40 & $16.7 \%$ & $1.19[0.99,1.44]$ & 2008 & & & $=$ & \\
\hline Abid et al & 55 & 60 & 47 & 60 & $17.5 \%$ & $1.17[1.00,1.36]$ & 2011 & & & $=$ & \\
\hline Mittal et al & 14 & 40 & 4 & 40 & $3.6 \%$ & $3.50[1.26,9.72]$ & 2011 & & & & \\
\hline Alvares de Silva et al & 2 & 28 & 1 & 35 & $0.8 \%$ & $2.50[0.24,26.17]$ & 2014 & & & & \\
\hline Sharma et al & 21 & 31 & 9 & 30 & $7.7 \%$ & $2.26[1.24,4.11]$ & 2016 & & & $\longrightarrow$ & \\
\hline Sidhu et al & 76 & 83 & 73 & 79 & $18.6 \%$ & $0.99[0.90,1.09]$ & 2018 & & & 1 & \\
\hline Total $(95 \% \mathrm{Cl})$ & & 424 & & 419 & $100.0 \%$ & $1.36[1.10,1.69]$ & & & & $\bullet$ & \\
\hline Total events & 302 & & 226 & & & & & & & & \\
\hline $\begin{array}{l}\text { Heterogeneity: } \operatorname{Tau}^{2}= \\
\text { Test for overall effect: }\end{array}$ & $\begin{array}{l}.06 ; \mathrm{Chi}^{2}= \\
=2.82(\mathrm{P}\end{array}$ & $\begin{array}{l}48.38 \\
=0.00\end{array}$ & $\begin{array}{l}\mathrm{df}=8(\mathrm{P} \\
5)\end{array}$ & $2<0.00$ & 0001): $1^{2}=$ & $83 \%$ & & 0.01 & 0.1 & $1 \quad 10$ & $\overrightarrow{100}$ \\
\hline
\end{tabular}

Forest plots indicating the pooled effect of LOLA versus placebo/ no intervention for improvement of mental state in all HE patients [19]. Abbreviations: RR: Risk Ratio, Cl: Confidence Interval

\section{C - Efficacy of LOLA versus placebo/no intervention: Prevention of OHE}

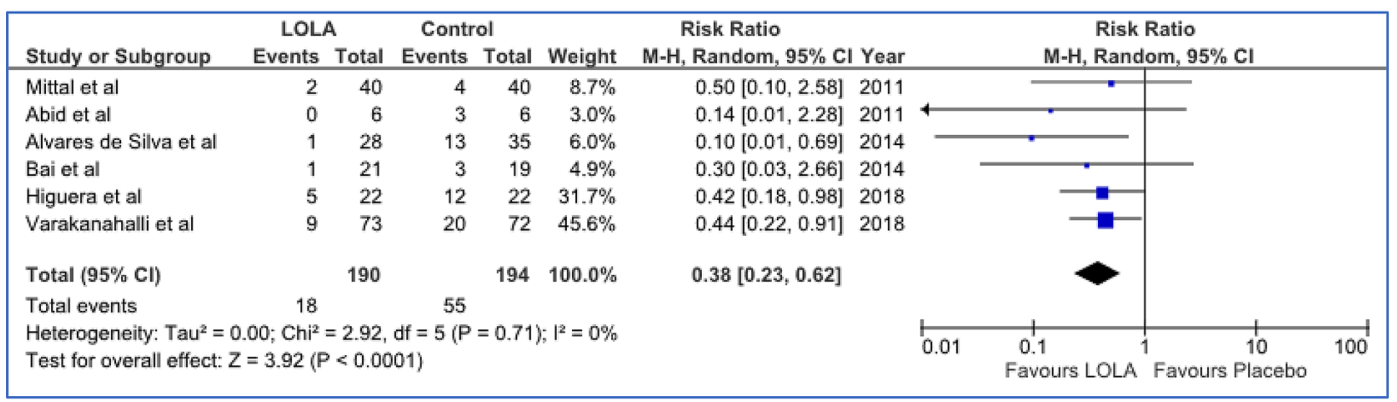


4Fig. 3 a Forest plot indicating the pooled effect of L-ornithine L-aspartate (LOLA) vs placebo/no intervention for the lowering of blood ammonia [19]. Efficacy of LOLA vs placebo/no intervention: $\mathrm{NH}_{3}$ lowering (all hepatic encephalopathy [HE] trials). b Forest plot indicating the pooled effect of LOLA vs placebo/no intervention for improvement of mental state in all patients with HE [19]. Efficacy of LOLA vs placebo/no intervention: mental state (all HE trials). c Forest plot for the efficacy of LOLA vs placebo/no intervention for the prevention of progression of minimal HE to overt HE (OHE) [10, $11,23]$, secondary OHE prophylaxis [24], primary OHE prophylaxis [25], or post-transjugular intrahepatic portosystemic shunt OHE prophylaxis [12] from results of the appropriate published randomised controlled trials [22]. Efficacy of LOLA vs placebo/no intervention: prevention of OHE. $C I$ confidence interval, $M-H$ MantelHaenszel test, $R R$ risk ratio, $S D$ standard deviation

converted via beta-oxidation to phenylacetate but, unlike SPA, GPB contains no sodium or adverse effects associated with SPA or SPB [38]. Rather it comprises three molecules of phenylbutyrate bonded to the molecule of glycerol in an esterified manner. Glycerol phenylbutyrate then becomes available for hydrolysis by pancreatic lipases, leading to the release of phenylbutyrate and glycerol. In a phase II trial in children with UCDs in which the efficacy of GPB was compared to SPB, the two agents were found to be at least equivalent in terms of ammonia control. Moreover, urinary phenylacetylglutamine levels were found to provide a clinically useful biomarker for dose selection and monitoring in patients with UCD [38]. Two years following this study in UCDs, results of a phase II RCT of GPB were described in patients with cirrhosis who had experienced two or more episodes of HE. Glycerol phenylbutyrate ( $6 \mathrm{~mL}$ twice daily) resulted in reductions of plasma ammonia and correlations with the number of HE events, time to first HE event and number of hospitalisations. Safety profiles were similar to placebo [39]. These findings were broadly confirmed in a subsequent Cochrane systematic review but the certainty (GRADE) of the evidence was judged to be "very low" [36].

\section{$5.4 O P$}

Ornithine phenylacetate (OP) has not been assessed for safety or efficacy in the treatment of UCDs. In the case of HE in cirrhosis, reports of the results of two RCTs have appeared. In the first trial, published in 2016, the efficacy of OP vs placebo was assessed in 38 consecutive patients within $24 \mathrm{~h}$ of an upper gastrointestinal bleed; the trial failed to demonstrate significant ammonia-lowering effects and there was no benefit for the incidence or severity of $\mathrm{HE}$ [40]. Some years later, a report on the efficacy and safety of OP administered in addition to standard of care according to current guidelines (which included the use of lactulose and/or rifaximin) for the treatment of OHE was published. No significant differences with regard to time to clinical improvement of $\mathrm{HE}$ based on ammonia measurements were observed between patients treated with OP compared to placebo [41]. A subsequent Cochrane review essentially confirming these findings reported evidence of OP-induced reduction of hyperammonaemia [36] in a single trial but again no improvements in mental state were observed and the trial was judged to have been of very low quality. The review compared a somewhat motley selection of agents for their safety and efficacy for the lowering of blood ammonia and improvement of HE status in patients with cirrhosis. The list included sodium benzoate, glycerol phenylbutyrate and OP as well as polyethylene glycol and AST-120. None of these agents was superior to lactulose for the lowering of blood ammonia and glycerol phenylbutyrate was the only agent to have a beneficial effect compared to placebo on both hyperammonaemia and HE. The quality of the evidence was judged to be uncertain with no confidence that any of the drugs were useful for the prevention or treatment of HE in people with cirrhosis, as the trials evaluated were very few in number and many did not provide sufficient data for inclusion in the analysis [36].

\section{Summary and Conclusions}

The lowering of circulating ammonia is pivotal for the prevention and treatment of $\mathrm{HE}$ in cirrhosis. Agents with the capacity to reduce ammonia absorption from the gut include non-absorbable disaccharides, antibiotics, probiotics, purgatives such as polyethylene glycol electrolyte solutions and a carbon microsphere preparation. Other agents have the metabolic capacity for the enhancement of active removal of ammonia by the liver in addition to the stimulation of ammonia removal by skeletal muscle.

L-Ornithine L-aspartate, a 1:1 mixture of the non-essential amino acids L-ornithine and L-aspartate, is effective for the lowering of circulating ammonia via multiple well-established mechanisms that include the optimisation of metabolic pathways (urea and glutamine synthesis) responsible for hepatic ammonia removal by residual periportal and perivenous hepatocytes in cirrhosis. L-Ornithine L-aspartate also has potential hepato-protective properties involving the production of the anti-oxidant glutathione and nitric oxide with the capacity to result in improved hepatic microcirculation. In addition, LOLA prevents cirrhosis-related sarcopenia, leading to improvements in ammonia removal by skeletal muscle.

Results of several independent systematic reviews each with a meta-analysis confirm that both the intravenous and oral formulations of LOLA are effective for the lowering of blood ammonia in patients with cirrhosis with concomitant improvements of mental state together with significant beneficial effects of LOLA for the prevention of OHE in the context of primary prophylaxis, secondary prophylaxis, for 
the reduction of the progression of MHE to OHE and for OHE occurring post-transjugular intrahepatic portosystemic shunt. L-Ornithine L-aspartate is reported to manifest a very good-to-excellent safety profile and its efficacy for ammonia lowering and improvement of mental state has been assessed as comparable or superior to alternative currently available agents such as non-absorbable disaccharides, antibiotics or probiotics as demonstrated in reports of the findings of recent network meta-analyses.

Reviews of the efficacy and safety of sodium benzoate, glycerol phenylbutyrate and ornithine phenylacetate concluded that none of these agents was superior to lactulose and neither were AST-120 or polyethylene glycol. Glycerol phenylbutyrate with its superior safety profile was the only agent shown to have a beneficial effect compared with placebo on hyperammonaemia and HE. The quality of the evidence in these trials was judged to be uncertain.

\section{Declarations}

Funding No funding was received for the preparation of this article.

Conflict of interest Roger F. Butterworth has received ad hoc payments for consulting and speaker fees from Salix Pharmaceuticals (USA), Umecrine Pharmaceuticals (Sweden) and Merz Pharmaceuticals (Germany).

Ethics approval Not applicable.

Consent to participate Not applicable.

Consent for publication Not applicable.

Availability of data and material Not applicable.

Code availability Not applicable.

Authors' contributions RFB was the sole author of the article.

Open Access This article is licensed under a Creative Commons Attribution-NonCommercial 4.0 International License, which permits any non-commercial use, sharing, adaptation, distribution and reproduction in any medium or format, as long as you give appropriate credit to the original author(s) and the source, provide a link to the Creative Commons licence, and indicate if changes were made. The images or other third party material in this article are included in the article's Creative Commons licence, unless indicated otherwise in a credit line to the material. If material is not included in the article's Creative Commons licence and your intended use is not permitted by statutory regulation or exceeds the permitted use, you will need to obtain permission directly from the copyright holder. To view a copy of this licence, visit http://creativecommons.org/licenses/by-nc/4.0/.

\section{References}

1. Montagnese S, Bajaj JS. Impact of hepatic encephalopathy in cirrhosis on quality-of-life Issues. Drugs. 2019;79(Suppl 1):11-6. https://doi.org/10.1007/s40265-018-1019-y (PMID:30706419;P MCID:PMC6416233).

2. Weissenborn K. Hepatic encephalopathy: definition, clinical grading and diagnostic principles. Drugs. 2019;79(Suppl 1):5-9. https://doi.org/10.1007/s40265-018-1018-z (PMID:30706420;P MCID:PMC6416238).

3. Butterworth RF. Hepatic encephalopathy in cirrhosis: pathology and pathophysiology. Drugs. 2019;79(Suppl 1):17-21. https:// doi.org/10.1007/s40265-018-1017-0 (PMID:30706423;PMCI D:PMC6416236).

4. Rahimi RS, Singal AG, Cuthbert JA, Rockey DC. Lactulose vs polyethylene glycol 3350-electrolyte solution for treatment of overt hepatic encephalopathy: the HELP randomized clinical trial. JAMA Intern Med. 2014;174(11):1727-33. https://doi.org/ 10.1001/jamainternmed.2014.4746 (PMID:25243839;PMCID: PMC5609454).

5. Pockros P, Hassanein T, Vierling J, Heuman D, Hillebrand D, Chojkier M, et al. Phase 2, multicenter, randomized study of AST120 (spherical carbon adsorbent) vs. lactulose in the treatment of low-grade hepatic encephalopathy (HE). J Hepatol. 2009;50:S434. https://doi.org/10.1016/S0168-8278(09)60107-0.

6. Kircheis G, Lüth S. Pharmacokinetic and pharmacodynamic properties of L-ornithine L-aspartate (LOLA) in hepatic encephalopathy. Drugs. 2019;79(Suppl 1):23-9. https://doi.org/10.1007/ s40265-018-1023-2 (PMID:30706424;PMCID:PMC6416235).

7. Butterworth RF. L-Ornithine L-aspartate: multimodal therapeutic agent for hyperammonemia and hepatic encephalopathy in cirrhosis. J Pharmacol Pharm Res. 2019;2(3):1-7.

8. Staedt U, Leweling H, Gladisch R, Kortsik C, Hagmüller E, Holm E. Effects of ornithine aspartate on plasma ammonia and plasma amino acids in patients with cirrhosis: a double-blind, randomized study using a four-fold crossover design. J Hepatol. 1993;19(3):424-30. https://doi.org/10.1016/s0168-8278(05) 80553-7 (PMID: 8151104).

9. Laubenberger J, Häussinger D, Bayer S, Gufler H, Hennig J, Langer M. Proton magnetic resonance spectroscopy of the brain in symptomatic and asymptomatic patients with liver cirrhosis. Gastroenterology. 1997;112(5):1610-6. https://doi.org/10.1016/ s0016-5085(97)70043-x (PMID: 9136840).

10. Abid S, Jafri W, Mumtaz K, Islam M, Abbas Z, Shah HA, et al. Efficacy of L-ornithine-L-aspartate as an adjuvant therapy in cirrhotic patients with hepatic encephalopathy. J Coll Physicians Surg Pak. 2011;21(11):666-71 (PMID: 22078345).

11. Alvares-da-Silva MR, de Araujo A, Vicenzi JR, da Silva GV, Oliveira FB, Schacher F, et al. Oral L-ornithine-L-aspartate in minimal hepatic encephalopathy: a randomized, double-blind, placebo-controlled trial. Hepatol Res. 2014;44(9):956-63. https://doi. org/10.1111/hepr.12235 (Epub 2013 Nov 11 PMID: 24033861).

12. Bai M, He C, Yin Z, Niu J, Wang Z, Qi X, et al. Randomised clinical trial: L-ornithine-L-aspartate reduces significantly the increase of venous ammonia concentration after TIPSS. Aliment Pharmacol Ther. 2014;40(1):63-71. https://doi.org/10.1111/apt. 12795 (Epub 2014 May 15 PMID: 24832463).

13. Butterworth RF, Grüngreiff K. L-Ornithine L-aspartate for the treatment of hepatic encephalopathy in cirrhosis: evidence for novel hepatoprotective mechanisms. JSM Liver Clin Res. 2019;3:5.

14. Canbay A, Sowa JP. L-Ornithine L-aspartate (LOLA) as a novel approach for therapy of non-alcoholic fatty liver disease. Drugs. 2019;79(Suppl 1):39-44. https://doi.org/10.1007/s40265-0181020-5 (PMID:30706422;PMCID:PMC6416231).

15. Desjardins P, Rao KV, Michalak A, Rose C, Butterworth RF. Effect of portacaval anastomosis on glutamine synthetase protein and gene expression in brain, liver and skeletal muscle. Metab Brain Dis. 1999;14(4):273-80. https://doi.org/10.1023/a:10207 41226752 (PMID: 10850554). 
16. Kumar A, Davuluri G, Silva RNE, Engelen MPKJ, Ten Have GAM, Prayson R, et al. Ammonia lowering reverses sarcopenia of cirrhosis by restoring skeletal muscle proteostasis. Hepatology. 2017;65(6):2045-58. https://doi.org/10.1002/hep.29107 (Epub 2017 Apr 28. PMID: 28195332; PMCID: PMC5444955).

17. Butterworth RF. L-Ornithine L-aspartate for the treatment of sarcopenia in chronic liver disease: the taming of a vicious cycle. Can J Gastroenterol Hepatol. 2019;28(2019):8182195. https:// doi.org/10.1155/2019/8182195 (PMID:31183339;PMCID: PMC6512019).

18. Butterworth RF, Kircheis G, Hilger N, McPhail MJW. Efficacy of L-ornithine L-aspartate for the treatment of hepatic encephalopathy and hyperammonemia in cirrhosis: systematic review and meta-analysis of randomized controlled trials. J Clin Exp Hepatol. 2018;8(3):301-13. https://doi.org/10.1016/j.jceh.2018.05.004.

19. Butterworth RF, McPhail MJW. L-Ornithine L-aspartate (LOLA) for hepatic encephalopathy in cirrhosis: results of randomized controlled trials and meta-analyses. Drugs. 2019;79(Suppl 1):317. https://doi.org/10.1007/s40265-018-1024-1 (PMID:30706425 ;PMCID:PMC6416237)

20. Goh ET, Stokes CS, Sidhu SS, Vilstrup H, Gluud LL, Morgan MY. L-Ornithine L-aspartate for prevention and treatment of hepatic encephalopathy in people with cirrhosis. Cochrane Database Syst Rev. 2018;5(5):CD012410. https://doi.org/10.1002/14651858. CD012410.pub2 (PMID: 29762873; PMCID: PMC6494563).

21. Dhiman RK, Thumburu KK, Verma N, Chopra M, Rathi S, Dutta $\mathrm{U}$, et al. Comparative efficacy of treatment options for minimal hepatic encephalopathy: a systematic review and network metaanalysis. Clin Gastroenterol Hepatol. 2020;18(4):800-12.e25. https://doi.org/10.1016/j.cgh.2019.08.047 (Epub 2019 Aug 30 PMID: 31476436).

22. Butterworth RF. Beneficial effects of L-ornithine L-aspartate for prevention of overt hepatic encephalopathy in patients with cirrhosis: a systematic review with meta-analysis. Metab Brain Dis. 2020;35(1):75-81. https://doi.org/10.1007/s11011-019-00463-8 (Epub 2019 Jul 23. PMID: 31338724; PMCID: PMC6974495).

23. Mittal VV, Sharma BC, Sharma P, Sarin SK. A randomized controlled trial comparing lactulose, probiotics, and L-ornithine L-aspartate in treatment of minimal hepatic encephalopathy. Eur J Gastroenterol Hepatol. 2011;23(8):725-32. https://doi.org/10. 1097/MEG.0b013e32834696f5 (PMID: 21646910).

24. Varakanahalli S, Sharma BC, Srivastava S, Sachdeva S, Dahale AS. Secondary prophylaxis of hepatic encephalopathy in cirrhosis of liver: a double-blind randomized controlled trial of L-ornithine L-aspartate versus placebo. Eur J Gastroenterol Hepatol. 2018;30(8):951-8. https://doi.org/10.1097/MEG.0000000000 001137(PMID: 29727385).

25. Higuera-de-la-Tijera F, Servín-Caamaño AI, Salas-Gordillo F, Pérez-Hernández JL, Abdo-Francis JM, Camacho-Aguilera J, et al. Primary Prophylaxis to Prevent the Development of Hepatic Encephalopathy in Cirrhotic Patients with Acute Variceal Bleeding. Can J Gastroenterol Hepatol. 2018;2018:3015891. https:// doi.org/10.1155/2018/3015891 (PMID: 30079329; PMCID: PMC6069577).

26. Fischer JE, Rosen HM, Ebeid AM, James JH, Keane JM, Soeters PB. The effect of normalization of plasma amino acids on hepatic encephalopathy in man. Surgery. 1976;80(1):77-91 (PMID: 818729).

27. Freund H, Yoshimura N, Fischer JE. Chronic hepatic encephalopathy: long-term therapy with a branched-chain amino-acidenriched elemental diet. JAMA. 1979;242(4):347-9. https://doi. org/10.1001/jama.242.4.347 (PMID: 36486).

28. Egberts EH, Schomerus H, Hamster W, Jürgens P. Branched chain amino acids in the treatment of latent portosystemic encephalopathy: a double-blind placebo-controlled crossover study.
Gastroenterology. 1985;88(4):887-95. https://doi.org/10.1016/ s0016-5085(85)80004-4 (PMID: 3882509).

29. Eriksson LS, Persson A, Wahren J. Branched-chain amino acids in the treatment of chronic hepatic encephalopathy. Gut. 1982;23(10):801-6. https://doi.org/10.1136/gut.23.10.801 (PMI D:6749604;PMCID:PMC1419824).

30. Marchesini G, Dioguardi FS, Bianchi GP, Zoli M, Bellati G, Roffi $\mathrm{L}$, et al. Long-term oral branched-chain aminoacid treatment in chronic hepatic encephalopathy: a randomized double-blind casein-controlled trial. The Italian Multicenter Study Group. J Hepatol. 1990;11(1):92-101. https://doi.org/10.1016/01688278(90)90278-y (PMID: 2204661).

31. Als-Nielsen B, Koretz RL, Kjaergard LL, Gluud C. Branchedchain amino acids for hepatic encephalopathy. Cochrane Database Syst Rev. 2003. https://doi.org/10.1002/14651858.CD001939 (Update in: Cochrane Database Syst Rev. 2015;2:CD001939. PMID: 12804416).

32. Marchesini G, Bianchi G, Merli M, Amodio P, Panella C, Loguercio C, Italian BCAA Study Group, et al. Nutritional supplementation with branched-chain amino acids in advanced cirrhosis: a double-blind, randomized trial. Gastroenterology. 2003;124(7):1792-801. https://doi.org/10.1016/s0016-5085(03) 00323-8 (PMID: 12806613).

33. Batshaw ML, Brusilow S, Waber L, Blom W, Brubakk AM, Burton BK, et al. Treatment of inborn errors of urea synthesis: activation of alternative pathways of waste nitrogen synthesis and excretion. N Engl J Med. 1982;306(23):1387-92. https://doi.org/ 10.1056/NEJM198206103062303 (PMID: 7078580).

34. De Las HJ, Aldámiz-Echevarría L, Martínez-Chantar ML, Delgado TC. An update on the use of benzoate, phenylacetate and phenylbutyrate ammonia scavengers for interrogating and modifying liver nitrogen metabolism and its implications in urea cycle disorders and liver disease. Expert Opin Drug Metab Toxicol. 2017;13(4):439-48. https://doi.org/10.1080/17425255.2017. 1262843 (Epub 2016 Nov 28. PMID: 27860485; PMCID: PMC5568887).

35. Sushma S, Dasarathy S, Tandon RK, Jain S, Gupta S, Bhist MS. Sodium benzoate in the treatment of acute hepatic encephalopathy: a double-blind randomized trial. Hepatology. 1992;16(1):138-44. https://doi.org/10.1002/hep.1840160123 (PMID: 1618465).

36. Zacharias HD, Zacharias AP, Gluud LL, Morgan MY. Pharmacotherapies that specifically target ammonia for the prevention and treatment of hepatic encephalopathy in adults with cirrhosis. Cochrane Database Syst Rev. 2019;6(6):012334. https://doi.org/ 10.1002/14651858.CD012334.pub2 (PMID: 31204790; PMCID: PMC6572872)

37. Honda S, Yamamoto K, Sekizuka M, Oshima Y, Nagai K, Hashimoto G, et al. Successful treatment of severe hyperammonemia using sodium phenylacetate powder prepared in hospital pharmacy. Biol Pharm Bull. 2002;25(9):1244-6. https://doi.org/10. 1248/bpb.25.1244 (PMID: 12230130).

38. Lichter-Konecki U, Diaz GA, Merritt JL, Feigenbaum A, Jomphe $\mathrm{C}$, Marier JF, et al. Ammonia control in children with urea cycle disorders (UCDs); phase 2 comparison of sodium phenylbutyrate and glycerol phenylbutyrate. Mol Genet Metab. 2011;103(4):3239. https://doi.org/10.1016/j.ymgme.2011.04.013 (Epub 2011 May 5. PMID: 21612962; PMCID: PMC4880058).

39. Rockey DC, Vierling JM, Mantry P, Ghabril M, Brown RS Jr, Alexeeva O, HALT-HE Study Group, et al. Randomized, double-blind, controlled study of glycerol phenylbutyrate in hepatic encephalopathy. Hepatology. 2014;59(3):1073-83. https://doi.org/ 10.1002/hep.26611 (PMID: 23847109; PMCID: PMC4237123).

40. Ventura-Cots M, Concepción M, Arranz JA, Simón-Talero M, Torrens M, Blanco-Grau A, et al. Impact of ornithine phenylacetate (OCR-002) in lowering plasma ammonia after upper gastrointestinal bleeding in cirrhotic patients. Therap Adv Gastroenterol. 
2016;9(6):823-35. https://doi.org/10.1177/1756283X16658252

(Epub 2016 Jul 26. PMID: 27803737; PMCID: PMC5076769).

41. Rahimi RS, Safadi R, Thabut D, Bhamidimarri KR, Pyrsopoulos

$\mathrm{N}$, Potthoff A, et al. Efficacy and safety of ornithine phenylacetate for treating overt hepatic encephalopathy in a randomized trial. Clin Gastroenterol Hepatol. 2020. https://doi.org/10.1016/j.cgh. 2020.10.019 (Epub ahead of print. PMID: 33069881). 